\title{
A ecocardiografia no diagnóstico da miocardiopatia não compactada: revisão narrativa
}

\author{
Echocardiography in the diagnosis of non-compacted cardiomyopathy: a narrative review \\ Ecocardiografía en el diagnóstico de miocardiopatía no compactada: revisión narrativa
}

Hudson Laert Machado de Miranda ${ }^{1 *}$, Marlucia do Nascimento Nobre1, João Marcos Bemfica Barbosa Ferreira ${ }^{1}$, Anne Elizabeth Andrade Sadala Marques ${ }^{1}$, Samira Corrêa Moreira ${ }^{1}$, Dênison Clark Corrêa de Miranda².

\section{RESUMO}

Objetivo: Conceituar e revisar a miocardiopatia não compactada como doença grave, sua apresentação clínica, suas relações com fatores genéticos e a importância do exame de ecocardiograma com doppler em seu diagnóstico. Revisão bibliográfica: $O$ miocárdio, junto com o sistema circulatório, inicia seu desenvolvimento na terceira semana gestacional, alterações genéticas na falha de compactação do miocárdio entre a quinta e a oitava semana, levam à persistência de trabeculações e recessos profundos, que se comunicam com a cavidade ventricular e geram espessamento do miocárdio em duas camadas distintas (compactada e não compactada). Os primeiros relatos desta patologia datam de 1926, sendo uma doença considerada rara, porém com fator genético considerável. Considerações finais: A miocardiopatia não compactada é uma doença muitas vezes subdiagnosticada por ser assintomática, porém um bom estudo ecocardiográfico, com profissional experiente e bem treinado quanto à questão é fundamental para seu diagnóstico, principalmente em pacientes jovens com história familiar da doença.

Palavras-chave: Miocardiopatia, Ecocardiograma, Insuficiência cardíaca.

\begin{abstract}
Objective: To conceptualize and review non-compacted cardiomyopathy as a serious disease, its clinical presentation, its relationship with genetic factors and the importance of the Doppler echocardiogram in its diagnosis. Bibliographic review: The myocardium, along with the circulatory system, starts its development in the third gestational week, genetic alterations in the failure of myocardial compaction between the fifth and eighth week, lead to the persistence of trabeculations and deep recesses, which communicate with the ventricular cavity and generate myocardial thickening in two distinct layers (compacted and non-compacted). The first reports of this pathology date back to 1926, being a disease considered rare, but with considerable genetic factor. Final considerations: Non-compacted cardiomyopathy is often a disease underdiagnosed for being asymptomatic, but a good echocardiographic study, with an experienced and well-trained professional in the matter, is essential for its diagnosis, especially in young patients with a family history of the disease.
\end{abstract}

Keywords: Cardiomyopathy, Echocardiogram, Heart failure.

\section{RESUMEN}

Objetivo: Conceptualizar y revisar la miocardiopatía no compactada como enfermedad grave, su presentación clínica, su relación con factores genéticos y la importancia del ecocardiograma Doppler en su diagnóstico. Revisión bibliográfica: El miocardio, junto con el sistema circulatorio, inicia su desarrollo en la

${ }^{1}$ Universidade Federal do Amazonas (UFAM), Manaus - AM. *E-mail: mirandahudsonpa@hotmail.com

2 Universidade Nilton Lins, Manaus - AM. 
tercera semana de gestación, alteraciones genéticas en el fallo de compactación miocárdica entre la quinta y octava semana, conducen a la persistencia de trabeculaciones y recesos profundos, que comunican con la cavidad ventricular y generan engrosamiento miocárdico en dos capas diferenciadas (compactada y no compactada). Los primeros reportes de esta patología se remontan a 1926, siendo una enfermedad considerada rara, pero con un factor genético considerable. Consideraciones finales: La miocardiopatía no compactada es a menudo una enfermedad infradiagnosticada por ser asintomática, pero un buen estudio ecocardiográfico, con un profesional experimentado y capacitado en la materia, es fundamental para su diagnóstico, especialmente en pacientes jóvenes con antecedentes familiares de la enfermedad.

Palabras clave: Miocardiopatía, Ecocardiograma, Insuficiencia cardíaca.

\section{INTRODUÇÃO}

Historicamente, a doença foi observada pela primeira vez em 1926 por Grant e colegas, quando realizou necrópsia de uma criança cardiopata e em 1975 por Dusek, que, também ao realizar necrópsias em crianças, notaram a persistência de um "miocárdio pós-natal esponjoso". Em 1984 Engberding e Bender, através do ecocardiograma bidimensional, evidenciaram a doença em pacientes vivos, porém apenas em 1990, Chin e colegas propuseram a nomenclatura atual, quando descreveram a presença de trabeculações proeminentes e numerosas, além de recessos intratrabeculares profundos. Dessa forma,entende-se que o aumento da pressão ou a isquemia miocárdica auxiliam na regressão dos sinosoides embrionários, fazendo com que haja persistência dos espaços intertrabeculares, havendo comunicação do ventrículo com a circulação embrionária (SOUSA DS, et al., 2018).

Contudo, apenas em 2006 a American Heart Association (AHA) a classificou como uma entidade separada. Assim, ficou caracterizada por duas camadas distintas da parede do ventrículo (geralmente 0 esquerdo): uma camada compactada externa e uma camada hipertrabeculada ou não compactada interna. A condição é caracterizada por disfunção progressiva do ventrículo, risco aumentado de arritmias ventriculares e tromboembolismo sistêmico (RODRIGUEZ-FANJUL J, et al., 2020).

A miocardiopatia não compactada é uma doença miocárdica heterogênea que pode levar a Insuficiência Cardíaca (IC), arritmias e/ou eventos embólicos (MAVROGENI SI, et al., 2020). A doença possui uma gama diversa de manifestações genotípicas e fenotípicas, mas sua morfologia característica consiste em um miocárdio de duas camadas com uma camada epicárdica compacta e trabeculações proeminentes que compreendem a camada endocárdica não compactada (CHEBROLU LH, et al., 2017).

Embora a miocardiopatia não compactada seja considerada uma condição rara, na verdade sua incidência e prevalência ainda são incertas. Foi reportada uma prevalência de $0,05 \%$ entre todos os exames ecocardiográficos de uma instituição de grande porte, mas em pacientes com IC sua foi descrita em cerca de 4\% (HOTTA VT, et al., 2017). Segundo estudos, ocorre em 0,81 por 100 lactentes/ano, em 0,12 casos por 100.000 crianças/ano e em adultos tem uma prevalência de $0,014 \%$, sendo que $12 \%$ e $50 \%$ dos pacientes com diagnóstico têm histórico familiar para a doença. Porém, tal estatística é subestimada, pois a maioria dos estudos foi realizado em hospitais terciários e em pacientes sintomáticos, sendo o sexo masculino mais acometido do que o sexo feminino (MENEGUZ-MORENO RA, et al., 2016; NASCIMENTO CU, et al., 2017; MARIA AS, et al., 2018).

Apesar de ter sido inicialmente descrita na população pediátrica, seu diagnóstico é cada vez mais comum em pacientes adultos, portanto sua manifestação pode ocorrer em qualquer fase da vida, já que as vezes fica assintomática por muitos anos. O prognóstico dos pacientes é determinado pelo grau e pela progressão da IC e pela presença de arritmias e de eventos tromboembólicos. Cerca de $60 \%$ dos pacientes sofrem morte súbita ou são submetidos a transplante cardíaco em um prazo de seis anos após o diagnóstico da doença. A ocorrência de complicações (como os eventos embólicos e arritmias) e de morte súbita parece ser consideravelmente menor em pacientes pediátricos, apesar da literatura não ter resposta adequada, quando comparados à subpopulação de adultos (BRONZATI L, et al., 2019). 
Pode ocorrer isoladamente ou em associação com outras miocardiopatias, outras síndromes complexas, bem como com distúrbios metabólicos e outras cardiopatias congênitas. Embora comumente acometa 0 Ventrículo Esquerdo (VE), também pode comprometer ambos os ventrículos ou apenas o Ventrículo Direito (VD) (HOTTA VT, et al., 2017). Na forma mais comum, que é quando afeta apenas o VE, também é conhecida como hipertrabeculação ventricular esquerda (FINSTERER J, et al., 2017; PAVANELLO R, 2017).

As manifestações clínicas em crianças e adultos é muito heterogênea, variando desde quadros assintomáticos até a tríade composta por IC congestiva, arritmias e tromboembolismo sistêmico. Fenômenos arritmogênicos, como fibrilação atrial e taquicardia ventricular, são mais comuns em adultos e estão relacionados à ocorrência de Acidente Vascular Cerebral (AVC) e embolia, além das taquicardias ventriculares graves estarem presentes em $20 \%$ dos casos (NASCIMENTO CU, et al., 2017; SOUSA DS, et al., 2018).

Nesse contexto de desenvolvimento, a miocardiopatia não compactada é uma doença rara, que resulta da falha de compactação do miocárdio entre a quinta e a oitava semanas do período embrionário, decorrente de alterações genéticas familiares ou esporádicas. Identifica-se a persistência de trabeculações e de recessos profundos, que fazem comunicação com a cavidade ventricular, gerando espessamento do miocárdio em tanto na camada compactada como na não compactada (MENEGUZ-MORENO RA, et al., 2016; NASCIMENTO CU, et al., 2017; MARIA AS, et al., 2018).

Do ponto de vista citogenético, já foram descritas mutações em vários genes humanos que codificam as proteínas sarcoméricas (envolvidas no processo de contração dos cardiomiócitos), do citoesqueleto e da membrana nuclear, incluindo: G4.5, TAZ, PRDM16, TNNT2, LDB3, MYBPC3, MYH7, ACTC1, TPM1, MIB1 e DTNA. Muitos destes genes estão associados a miocardiopatia dilatada ou hipertrófica. Também foram identificadas três mutações no gene HCN4, quando analisada uma família holandesa que apresentava bradicardia sinusal e miocardiomiopatia não compactada simultaneamente, que diminuem a corrente If (corrente elétrica que possui papel fundamental na regulação do ritmo cardíaco) nas células sinoatriais, causando bradicardia (MENEGUZ-MORENO RA, et al., 2016; CARLESSO VB, et al., 2018).

Devido às complicações que pode causar, seu diagnóstico e tratamento precoces são de extrema importância, sendo a ecocardiografia a modalidade de imagem de primeira linha. Além disso, a avaliação genética e o aconselhamento são cruciais para a avaliação de risco individual e o planejamento familiar (MAVROGENI SI, et al., 2020).

Dessa forma, este trabalho teve por objetivo demonstrar e revisar a complexidade dessa doença, suas manifestações e alertar que pode ser um diagnóstico inesperado, porém de fácil percepção durante um exame simples de ecocardiograma transtorácico, que é o foco desta revisão.

\section{REVISÃO BIBLIOGRÁFICA}

\section{Anatomia Miocárdica}

O desenvolvimento macroscópico das câmaras cardíacas, atriais e ventriculares ocorre no tubo cardíaco linear inicial, liso, de duas camadas, por meio de um padrão de alterações morfológicas complexas conhecido como maturação da câmara cardíaca. A trabeculação, juntamente com o desenvolvimento do sistema de condução e espessamento do miocárdio, devidamente compactado, é um dos processos de maturação da câmara cardíaca, com a trabeculação consistindo em três estágios sequenciais que, em última instância, resultam na fusão com a camada de miocárdio compactada e progressão da parede ventricular espessa e madura. As trabeculações são protuberâncias da parede ventricular em desenvolvimento para o lúmen ventricular, com uma estrutura idêntica ao tubo cardíaco embrionário e células miocárdicas revestidas pela camada endotelial do endocárdio (WENGROFSKY P, et al., 2019).

A trabeculação é seguida por remodelação, também conhecida como consolidação ou compactação, durante a qual as extremidades distais das trabéculas param de crescer e as extremidades proximais ou da base se espessam e colapsam para se parecerem visualmente com a camada miocárdica compactada subjacente, com os espaços entre os botões trabeculares se desenvolvendo em capilares. Defeitos em 
qualquer estágio nas junções de emergência, trabeculação e remodelação da maturação da câmara cardíaca podem resultar na persistência de trabeculações e impedir a proliferação adequada do miocárdio compactado (SANTOS PPD, et al., 2021).

O miocárdio é composto por três tipos de cardiomiócitos, que são células especializadas (as fibras musculares do coração) que envolvem as câmaras do coração, formando uma espiral complexa, inserindose no esqueleto cardíaco fibroso. Esses três tipos possuem funções diferentes: 1) contráteis, que são os que bombeiam sangue através da circulação; 2) mioendócrinos, que produzem o fator natriurético atrial; 3) nodais, que se localizam no nodo sinoatrial e atrioventricular, sendo responsáveis por controlar a contração rítmica do coração. A unidade contrátil cardíaca é chamada de sarcômero, que é o responsável pela aparência estriada das fibras, que corresponde a um arranjo de filamentos espessos que são compostos por miosina e filamentos finos de actina, troponina e tropomiosina (FREITAS DMO, et al., 2018).

Os cardiomiócitos na camada subepicárdica são menos diferenciados do que os das regiões trabeculadas e, embora a absorção de oxigênio e nutrientes seja limitada pela difusão devido às trabéculas e camadas miocárdicas internas, o crescimento do miocárdio compacto é impulsionado por um espessamento mais lento e aposição de camadas cada vez mais compactas de cardiomiócitos. A proliferação do miocárdio compactado são aceleradas pelo fornecimento de oxigênio e nutrientes via vascularização coronariana originada do epicárdio, aumentando ainda mais a contribuição da camada compactada para a massa miocárdica ventricular total. Embora a não compactação das trabeculações ventriculares possa surgir de qualquer distúrbio ao longo de todo o processo de maturação das câmaras cardíacas, acredita-se que a falha na etapa de remodelação com consolidação resulta em não compactação (WENGROFSKY P, et al., 2019).

\section{Fatores Genéticos}

Acredita-se que a miocardiopatia não compactada resulte do fracasso da fase final da compactação miocárdica embriogênica, com aumento da evidência de um componente genético na forma de mutações em genes que codificam proteínas sarcoméricas, citoesqueléticas e de membrana nuclear tornando-se aparentes. Este último é refletido pela classificação da miocardiopatia não compactada pela American Heart Association e pelo European Society of Cardiology Working Group on Myocardial and Pericardial Diseases, embora com diferenças quanto à extensão do reconhecimento de um gene primário de etiologia oposta à ocorrência familiar não classificada (MAVROGENI SI, et al., 2020; BREEN CJ, et al., 2019).

Estima-se que em $50 \%$ dos pacientes com esta patologia exista uma causa genética, porque há mutação em gene de cardiomiopatia e/ou pelo menos um membro da família apresenta cardiomiopatia não isquêmica, sendo estas alterações mais comuns em genes do sarcômero. Em 15\% dos pacientes, a doença familiar ocorre sem mutação. Entre os genes da miocardiopatia não compactada, os mais prevalentes são MYH7, MYBPC3 e TTN. Temos ainda, o gene FKBP12, relacionado à liberação de cálcio do retículo sarcoplasmático pelo receptor RyR2, que, quando alterado, gera displasia arritmogênica do ventrículo direito. Alterações nos genes LAMINA A/C, presente no cromossomo 1q22, nos fatores de transcrição NKX2.5 e TBX5, no cromossomo $11 \mathrm{p} 15$, deleção distal do cromossomo 5 , também foram associadas à miocardiopatia não compactada (VAN WANING JI, et al., 2018; VAN WANING JI, et al., 2019; WENGROFSKY P, et al., 2019).

\section{Manifestações Clínicas}

Os sintomas clínicos variam de manifestações pré-natais graves a cardiomiopatia sintomática que se apresenta em adultos (VAN WANING JI, et al., 2018). É uma doença que costuma evoluir progressivamente com disfunção sistólica e diastólica culminando com IC congestiva. A disfunção ventricular esquerda foi constatada em até $84 \%$ dos pacientes em geral, a fibrilação atrial em aproximadamente $25 \%$ dos pacientes adultos, enquanto as taquiarritmias ventriculares são observadas em até $47 \%$ de todos os pacientes. Eventos tromboembólicos, entre eles o acidente vascular encefálico, a embolia pulmonar, o ataque isquêmico transitório e a isquemia mesentérica possuem incidência de até 38\% (HOTTA VT, et al., 2017; FRIEDMANN $A A, 2018)$.

Anormalidades eletrocardiográficas podem ser verificadas em até $90 \%$ dos portadores da doença, contudo, são alterações inespecíficas. Entre os achados mais comuns estão os atrasos na condução 
intraventricular, alterações da repolarização ventricular, hipertrofia ventricular esquerda e achados de síndrome de Wolff-Parkinson-White. O tromboembolismo é uma das principais manifestações da doença, mas outras complicações associadas consideradas mais preocupantes são o infarto cerebral, a insuficiência cardíaca e a morte cardíaca súbita (WENGROFSKY P, et al., 2019; HOTTA VT, et al., 2017).

\section{Diagnóstico Pelo Ecocardiograma Transtorácico}

Por definição, o ecocardiograma transtorácico é o mais utilizado na Cardiologia, dada sua disponibilidade, fácil execução e demonstrar, em tempo real, o que está ocorrendo com o coração. No âmbito da miocardiopatia não compactada é o exame diagnóstico e de seguimento clínico (SCUDERI C, et al., 2019). É composto por uma combinação integrada de imagens bidimensionais, Doppler espectral pulsado e contínuo, Doppler codificado em cor e, normalmente, modo $\mathrm{M}$ e Doppler tecidual, sendo o exame completo definido pela avaliação morfológica e funcional de todas as câmaras cardíacas, válvulas e grandes vasos, utilizando diferentes planos de imagem (SAMPAIO F, et al., 2018).

Um exame ecocardiográfico transtorácico completo deve incluir tanto uma avaliação morfológica como funcional de todas as câmaras cardíacas, assim como válvulas e grandes vasos, utilizando diferentes planos de imagem. Isto envolve medições sobre as imagens bidimensionais, de Modo $\mathrm{M}$ e de Doppler espectral e tecidular, bem como a quantificação da função sistólica e diastólica, realização do cálculo dos orifícios valvulares estenóticos e regurgitantes além de estimativas de pressões de enchimento e pressão arterial pulmonar (AFONSO TR, et al., 2016).

Os critérios ecocardiográficos de Jenni e Chin são os mais comumente usados para a identificação de não compactação. Ambos contam com uma relação de camada não compactada para camada compactada de pelo menos dois. Os critérios de Chin usam as medidas dos componentes da parede do VE no final da diástole, enquanto os critérios de Jenni consideram as medições feitas no final da sístole (RODRIGUEZFANJUL J, et al., 2020).

Segundo Nascimento CU, et al. (2017) os critérios ecocardiográficos para diagnóstico de miocardiopatia não compactada isolada são a visualização de trabéculas e recessos intratrabeculares profundos, apresentando espessamento $>2$ vezes da camada não compactada endocárdica em comparação com a camada compactada do miocárdio, com predomínio nas regiões apicais, lateral média e inferior média do VE esquerdo, ter presença de recessos intratrabeculares com perfusão direta pelo ventrículo e ausência de anormalidades cardíacas coexistentes.

O diagnóstico de miocardiopatia não compactada é feito convencionalmente com imagem ecocardiográfica bidimensional (2DE), que permite demonstrar a presença de trabeculações proeminentes com recessos intertrabeculares profundos em continuidade com a cavidade ventricular (e não com o sistema de circulação coronariana epicárdica) e uma aparência típica de duas camadas no miocárdio, camada epicárdica compacta mais fina e não compactada, além da camada endocárdica não compactada mais espessa (CHEBROLU LH, et al., 2017).

A avaliação da função miocárdica regional usando 2DE foi estabelecida há muito tempo usando o modelo de 17 segmentos. A quantificação ecocardiográfica da função miocárdica regional é atualmente baseada em técnicas de Doppler Tecidual (TDI) ou ecocardiográfica com Speckle Tracking (STE) e também, mais recentemente, com a tecnologia 3D Speckle Tracking (3DSTE) (COELHO MR, et al., 2020).

A aplicação da ecocardiografia speckle tracking permite a identificação da dinâmica de contração rotacional anormal do VE, ou seja, uma rotação dos segmentos basal e apical na mesma direção e, portanto, ausência de torção ventricular esquerda fisiológica (sentido horário na base e anti-horário no ápice). A ecocardiografia $3 \mathrm{D}$ em tempo real é outro método recentemente introduzido, que permite uma quantificação mais precisa do número de trabeculações e da massa ventricular. A ecocardiografia com contraste pode aumentar a sensibilidade da ecocardiografia para o diagnóstico da doença, assim como pode "colorir" o miocárdio (Doppler tecidual) (FINSTERER J, et al., 2017).

Os aspectos pertinentes da avaliação funcional regional de miocardiopatia não compactada podem ser resumidos como: 1) A distribuição de segmentos hipocinéticos (segmentos apicais > basais), especialmente 
em pacientes com função sistólica reduzida em pacientes com miocardiopatia não compactada, concordaria com o desenvolvimento anatômico pelo qual a compactação da camada trabecular ocorre da base ao ápice, da parede septal à lateral e do epicárdio a endocárdio explicando a preservação relativa dos segmentos basais; 2) A distribuição dos segmentos hipocinéticos em pacientes com Fração de Ejeção (FE) preservada (segmentos apicais > basais), além da presença de malha ou hipertrabeculação nos segmentos lateral e apical, auxilia no diagnóstico de miocardiopatia não compactada; 3) A contribuição diferencial de segmentos compactados e não compactados para a função sistólica geral no miocardiopatia não compactada (CHEBROLU LH, et al., 2017).

Além disso, um estudo volumétrico com ecocardiografia tridimensional em tempo real (RT3DE) também demonstrou que os segmentos não compactado e no compactado do VE tinham volumes regionais comparativamente aumentados e função sistólica reduzida, sugerindo que a disfunção sistólica do VE na miocardiopatia não compactada não está confinada aos segmentos não compactados (NEMES A, et al., 2018).

Segundo Finsterer J, et al. (2017), as desvantagens da ecocardiografia transtorácica incluem a imprecisão de planos de imagem fora do eixo ou oblíquos e os desafios de avaliar o ápice. No entanto, ter a vista apical de quatro câmaras projetada de cabeça para baixo ou do lado direito para cima pode ajudar a visualizar o ápice. Essas desvantagens podem ser parcialmente superadas com o uso da ecocardiografia transesofágica, que em alguns casos pode permitir o diagnóstico de miocardiopatia não compactada que não foi detectada na ecocardiografia transtorácica. A hipertrabeculação ou não compactação do ventrículo direito pode não ser diagnosticada, pois os limites de referência para essa condição ainda não estão disponíveis, mas a identificação de trabeculações qualitativamente grosseiras no ápice do ventrículo direito deve ser estabelecida.

\section{Tratamento Dos Pacientes}

Os dados disponíveis sobre as opções de tratamento para miocardiopatia não compactada são limitados, mas seus pontos cruciais são a terapia para IC (incluindo transplante cardíaco), terapia antiarrítmica (incluindo ablação e implantação de desfibrilador ou cardioversor) e anticoagulante oral (FINSTERER J, et al., 2017). O tratamento tem como objetivo compensar a IC e prevenir a ocorrência de arritmias e de tromboembolismo. Em casos de insuficiência refratária, a única opção é o transplante cardíaco (FRIEDMANN AA, 2018).

Como o tromboembolismo é uma das principais manifestações da doença, algumas diretrizes, incluindo a do Brasil, recomendam uso de anticoagulante nos pacientes que possuam função sistólica diminuída com fração de ejeção inferior a 40\%, histórico de tromboembolismo ou fibrilação atrial, assim como o uso de ácido acetilsalicílico em pacientes que estejam assintomáticos e com função sistólica normal (HOTTA VT, et al., 2017).

Levando-se em consideração, que a história natural da doença leva à IC, o uso de betabloqueadores e inibidores da conversão de angiotensinogênio também são indicados. Em casos de arritmia ventricular sintomática e função sistólica prejudicada, se indica o uso de agentes antiarrítmicos ou de desfibriladores cardíacos implantáveis para prevenir um evento sustentado e de arritmia potencialmente letal, conforme as diretrizes internacionais (POFFO MR, et al., 2017).

\section{CONSIDERAÇÕES FINAIS}

A miocardiopatia não compactada é uma doença que está presente no dia a dia, porém subdiagnosticada. Com o avanço da tecnologia, os aparelhos de ecocardiograma com doppler apresentam melhores definições e qualidades de imagem, dessa forma, somando-se a experiência do ecocardiografista e da melhor percepção do paciente baseado nos achados clínicos como arritmias, tromboembolismo e IC, deve-se sempre lembrar dessa patologia, para iniciar o tratamento precoce, uma vez que, quadros de morte súbita estão associados. Além disso, uma vez que o paciente é diagnosticado, uma triagem familiar rigorosa deve ser realizada, pois, em $50 \%$ dos casos, algum parente próximo pode apresentar a doença, mesmo estando assintomático. 


\section{REFERÊNCIAS}

1. AFONSO TR, et al. Influência do Posicionamento do Paciente sobre a qualidade das imagens ecocardiográficas em exames realizados no Leito. Arq Bras Cardiol: ImagemCardiovas [Internet], 2016; 29(4); 112-7.

2. BREEN CJ, et al. ECG interpretation skill acquisition: A review of learning, teaching and assessment. Journal of electrocardiology, 2019; 1(1): 1-5.

3. BRONZATI L, et al. Acidente Vascular Encefálico Isquêmico Reincidente Em Paciente Sem Risco Epidemiológico. Revista Uningá, 2019; 56(4): 98-103.

4. CALESSO VB, et al. Cardiomiopatia não compactada: doença do nó sinusal como manifestação inicial tratada com marcapasso. RELAMPA, 2018; $31(2):$ 73-5.

5. CHEBROLU LH, et al. Noncompaction cardiomyopathy: The role of advanced multimodality imaging techniques in diagnosis and assessment. Echocardiography, 2017; 34(2): 279-89.

6. COELHO MR, et al. Avaliação da deformação miocárdica pela ecocardiografia feature tracking em gatos com defeito perimembranoso do septo ventricular. Arquivo Brasileiro de Medicina Veterinária e Zootecnia, 2020; 72(3): 807-813.

7. FINSTERER J, et al. Left ventricular noncompaction cardiomyopathy: cardiac, neuromuscular, and genetic factors. Nature reviews. Cardiology, 2017; 14(4): 224-37.

8. FREITAS DMO, et. al. Síndrome coronariana aguda: Parte 1 - Abordagem estrutural e Fisiopatologia. Revista Eletrônica Acervo Saúde, 2018; 15(Supl):1928-34.

9. FRIEDMANN AA. Cardiomiopatia não compactada. Diagnóstico e Tratamento, 2018; 23(1): 15-8.

10. HOTTA VT, et al. Limitações no diagnóstico de miocárdio não compactado pela ecocardiografia. Arquivos Brasileiros de Cardiologia, 2017; 109(5): 483-88.

11. MARIA AS, et al. Taquicardia ventricular idiopática em portador de miocárdio não compactado. RELAMPA, 2018; $31(1): 23-6$.

12. MAVROGENI SI, et al. The pivotal role of cardiovascular imaging in the identification and risk stratification of noncompaction cardiomyopathy patients. Heart Failure Reviews, 2020; 25(6), 1007-15.

13. MENEGUZ-MORENO RA, et al. Revista Portuguesa de Cardiologia, 2016; 35(3): 185.e1-4.

14. NASCIMENTO CU, et. al. Miocardiopatia não compactada: relato de caso. Arquivos de Ciências da Saúde, 2017; 24(1): 3-6.

15. NEMES A, et al. Análise da Deformação Atrial Direita na Amiloidose Cardíaca-Resultados de Ecocardiografia Tridimensional de Speckle-Tracking do Estudo MAGYAR-Path. Arquivos Brasileiros de Cardiologia, 2018; 111(1): 384391

16. PAVANELLO R. Terapia coagulante nas miocardiopatias. Rev. Soc. Cardiol. Estado de Säo Paulo, $2017 ; 1$ (1): f-234.

17. POFFO MR, et al. Perfil dos pacientes internados por insuficiência cardíaca em hospital terciário. International Journal of Cardiovascular Sciences, 2017; 30(3): 189-198.

18. RODRIGUEZ-FANJUL J, et al. Neonatal non-compacted cardiomyopathy: predictors of poor outcome. Pediatric Cardiology, 2020; 41(1): 175-80.

19. SAMPAIO F, et al. Documento de consenso e recomendações para a realização de ecocardiografia transtorácica em Portugal. Revista Portuguesa de Cardiologia, 2018; 37(8): 637-44.

20. SANTOS PPD, et al. Suplementação de Vitamina D Induz Remodelação Cardíaca em Ratos: Associação com a Proteína de Interação com a Tiorredoxina e a Tiorredoxina. Arquivos Bras de Cardiologia, 2021; 116(1): $970-978$.

21. SCUDERI C, et al. Miocardiopatia não compactada: um relato de caso. FAG Journal of Health, 2019; 1(4): 124-9.

22. SOUSA DS, et. al. Miocardiopatia não compactada - uma revisão da literatura. Revista de Patologia do Tocantins, 2018; 5(2): 74-8.

23. VAN WANING JI, et al. Genetics, clinical features, and long-term outcome of noncompaction cardiomyopathy. Journal of the American College of Cardiology, 2018; 71(7): 711-22.

24. VAN WANING JI, et al. Systematic review of genotype-phenotype correlations in noncompaction cardiomyopathy. Journal of the American Heart Association, 2019; 8(23): e012993.

25. WENGROFSKY $P$, et al. Left ventricular trabeculation and noncompaction cardiomyopathy: a review. EC Clinical and Experimental Anatomy, 2019; 2(6): 267-83. 